\title{
Berichtigung zu der Arbeit: On Algebraic Curves over Real Closed Fields
}

Math. Z. 150, 49-70 (1976)

Manfred Knebusch

Fachbereich Mathematik der Universität, D-8400 Regensburg, Bundesrepublik Deutschland

In Appendix B der Arbeit wurde der Satz aufgestellt, daß für jedes Differential $\omega$ der vollständigen glatten Kurve $X$ über einen reell abgeschlossenen Körper $k$ die Summe der Residuen in den Punkten einer Komponente $\gamma_{i}$ der Menge der reellen Punkte von $X$ verschwindet. Dieser Satz ist falsch, wie etwa das Differential $\omega=\left(1+x^{2}\right)^{-1} x d x$ auf der projektiven Geraden zeigt, mit $x$ eine Erzeugende des Funktionenkörpers. Der Fehler im „Beweis“ des Satzes beruht auf einer fehlerhaften Anwendung des klassischen Residuensatzes in der letzten Zeile der Arbeit.

Ich wurde auf diesen Fehler von Herrn W.D. Geyer aufmerksam gemacht. Der Fehler berührt die anderen Resultate der Arbeit nicht, auch nicht die von Teil II (Math. Z. 151, 1976), weil Appendix B nirgends benutzt wird. 